\title{
Neoadjuvant Chemotherapy with Docetaxel, Carboplatin and Weekly Trastuzumab Is Active in HER2-Positive Early Breast Cancer: Results after a Median Follow-Up of over 4 Years
}

\author{
Hans-Christian Kolberg ${ }^{a} \quad$ Leyla Akpolat-Basci $^{a} \quad$ Miltiades Stephanou $^{a} \quad$ Bahriye Aktas $^{b}$ \\ Carla Verena Hannig ${ }^{c}$ Cornelia Liedtke $^{\mathrm{d}}$ \\ ${ }^{a}$ Klinik für Gynäkologie und Geburtshilfe, Marienhospital Bottrop, Bottrop, Germany;

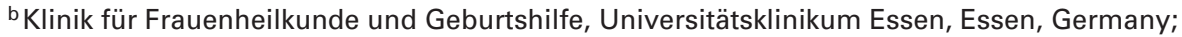 \\ ${ }^{c}$ Schwerpunktpraxis für Hämatologie und Onkologie, Bottrop, Germany; \\ ${ }^{\mathrm{d}}$ Klinik für Frauenheilkunde und Geburtshilfe, Universitätsklinikum Schleswig-Holstein Campus Lübeck, Lübeck, Germany
}

\section{Keywords}

Neoadjuvant therapy - HER2 positive - Breast cancer . Anthracycline free $\cdot \mathrm{TCH}$

\section{Summary}

Introduction: Most patients with HER2-positive breast cancer receive chemotherapy and trastuzumab. Data from adjuvant trials have shown that the combination of docetaxel, carboplatin and weekly trastuzumab (TCH) is well tolerated and as effective as anthracycline-containing regimes. Previous investigations on neoadjuvant treatment with taxanes, platinum salts and trastuzumab showed pathological complete remission (pCR) rates between 43.3 and $76 \%$. To date, the longest published follow-up in this indication is 3 years. Here we present 4-year follow-up data for a cohort of 78 patients treated with neoadjuvant TCH. Methods: Between 2009 and 2014 we treated 78 patients with operable HER2positive breast cancer with a neoadjuvant schedule of docetaxel $\left(75 \mathrm{mg} / \mathrm{m}^{2}\right.$ ) and carboplatin (AUC 6) every 3 weeks $(\mathrm{q} 3 \mathrm{w})$ and trastuzumab $(4 \mathrm{mg} / \mathrm{kg}$ loading dose then $2 \mathrm{mg} / \mathrm{kg}$ ) q1w. Lymph node involvement was verified by sentinel lymph node or core-cut biopsy. Patients were diagnosed at a mean age of 55.5 years; $65.4 \%$ had hormone receptor-positive tumors, $34.6 \%$ presented with grade 3 disease and $51.3 \%$ of patients were node positive. Patients were monitored every 2 cycles by ul- trasound. After 6 cycles of chemotherapy all patients had surgery. Axillary dissection was performed in case of positive lymph node status prior to $\mathrm{TCH}$. After surgery, trastuzumab was continued q3w up to 1 year. Results: No grade III/IV toxicities occurred and no case of congestive heart failure was observed. Neither dose modifications nor dose delays were necessary. 34 of the 78 patients $(43.6 \%)$ achieved a pCR, 27 of the 40 node-positive patients (67.5\%) experienced nodal conversion. After a median follow up of 48.5 months the disease-free survival (DFS) was $84.6 \%$, the distant disease-free survival (DDFS) was $87.2 \%$ and the overall survival (OS) was $91 \%$. Only T stage and nodal status at baseline were found to be significantly associated with survival estimates. Conclusion: The anthracycline-free regimen $\mathrm{TCH}$ is effective and safe in the neoadjuvant therapy of HER2-positive breast cancer, yielding DFS, DDFS and OS probabilities comparable to the results of adjuvant trials. Our data support the use of $\mathrm{TCH}$ as a neoadjuvant therapy regimen for patients with HER2positive breast cancer. They also strongly encourage the use of taxanes and platinum salts as the chemotherapy backbone in studies investigating dual blockade with trastuzumab and pertuzumab in the neoadjuvant setting.

(c) 2016 S. Karger GmbH, Freiburg

\section{KARGER}

() 2016 S. Karger GmbH, Freiburg 


\section{Introduction}

The prognosis for HER2-positive breast cancer is worse than the prognosis for HER2-negative tumors. In the adjuvant setting, this poor prognosis has been significantly improved by anti-HER2 therapy with trastuzumab $[1,2]$. In the neoadjuvant setting, the addition of a HER2-targeted therapy to chemotherapy has resulted in an increased rate of pathological complete remission (pCR), indicating that a positive HER2 status is a predictive marker for response to neoadjuvant chemotherapy. The German GeparQuattro trial demonstrated that the addition of trastuzumab to neoadjuvant chemotherapy for HER2-positive breast cancer was associated with an increase in pCR rate from 15.7 to $31.7 \%$ [3]. These beneficial pCR rates are translating into improved survival probabilities. A recently published pooled analysis of 12 neoadjuvant studies [4] showed that pCR (defined as ypT0 ypN0 or ypTis ypN0) is associated with improved overall survival (OS). In the analysis of the total cohort, irrespective of biological subtypes, a significant benefit for event-free survival (EFS) (hazard ratio (HR) 0.48) and OS (HR 0.36) was seen. In the HER2-positive/hormone receptor-negative subgroup, achieving a pCR was associated with a significant advantage in terms of EFS (HR 0.25).

Based upon concerns regarding cardiotoxicity, anthracyclinefree chemotherapy backbones for trastuzumab have been explored. In the adjuvant setting, the BCIRG-006 trial compared an anthracycline-containing regime with or without the addition of trastuzumab with the anthracycline-free combination of docetaxel, carboplatin and trastuzumab (TCH). As expected, patients in the study arm without trastuzumab did worse than in the 2 arms containing trastuzumab. However, there was no significant difference between the 2 groups receiving trastuzumab, no matter whether they received an anthracycline and a taxane or carboplatin and a taxane, whereas the rates of serious congestive heart failures favored the TCH arm [2].

The pCR rates achieved in the neoadjuvant setting by combinations of taxanes, platinum salts and trastuzumab ranged from 43.3 to $76 \%$ [5-8]. Our own workgroup presented a pCR of $64 \%$ in a cohort treated with $\mathrm{TCH}$, the regimen used in the BCIRG-006 trial [9]. To date, the longest published follow-up for patients receiving the combination of a taxane, a platinum salt and trastuzumab as a neoadjuvant therapy for HER2neu-positive breast cancer has been 3 years and that study showed a recurrence-free survival rate of $71 \%$ and an OS rate of $86 \%$ [10].

Here we report the survival variables of a cohort with a median follow-up of over 4 years in this indication.

\section{Material and Methods}

\section{Patient Characteristics}

Between 2009 and 2014 we treated 78 patients with operable HER2-positive breast cancer with a neoadjuvant schedule of docetaxel $\left(75 \mathrm{mg} / \mathrm{m}^{2}\right)$ every 3 weeks (q3w), carboplatin (AUC 6) q3w and trastuzumab (4 mg/kg loading dose then $2 \mathrm{mg} / \mathrm{kg}$ ) q1w, i.e. the regimen used in the BCIRG-006 trial [2]. The mean age of the patients was 55.5 years (range 23-80 years).
Table 1. Patient characteristics at baseline $(\mathrm{n}=78)$

\begin{tabular}{lc}
\hline & $\mathrm{n}(\%)$ \\
\hline Grading & $1(1.3)$ \\
G1 & $50(64.1)$ \\
G2 & $27(34.6)$ \\
G3 & \\
Hormone receptor status & $51(65.4)$ \\
Positive & $27(34.6)$ \\
Negative & \\
T stage at diagnosis & $38(48.7)$ \\
T1 & $33(42.3)$ \\
T2 & $1(1.3)$ \\
T3 & $6(7.7)$ \\
T4 & \\
Nodal status at diagnosis & $38(48.7)$ \\
Negative & $40(51.3)$ \\
Positive & \\
Menopausal status & $21(26.9)$ \\
Premenopausal & $57(73.1)$ \\
Postmenopausal &
\end{tabular}

Table 2. Patient characteristics with a statistically significant association with survival variables

\begin{tabular}{llll}
\hline & \multicolumn{2}{l}{ Survival variable } & \\
\cline { 2 - 4 } & DDFS & DFS & OS \\
\hline Tumor stage at baseline & & & \\
T1, \% & 100 & 97.4 & 100 \\
Other, \% & 75 & 72.5 & 82.5 \\
Log rank $\mathrm{p}$ & 0.0001 & 0.001 & 0.006 \\
Nodal status at baseline & & & \\
Negative, \% & 97.4 & 94.7 & \\
Positive, \% & 77.5 & 75.0 & \\
Log rank $\mathrm{p}$ & 0.012 & 0.023 & \\
\hline
\end{tabular}

DDFS = Distant disease-free survival, DFS = disease-free survival, OS = overall survival.

All patients received a staging prior to the start of neoadjuvant therapy for lung, liver and bone metastases and were all found to be negative. Patients with clinically negative lymph nodes received a sentinel lymph node biopsy (SLNB) prior to neoadjuvant therapy. Lymph node involvement was verified by SLNB or core-cut biopsy. All tumors were marked with titanium clips before neoadjuvant therapy. Patient characteristics are shown in table 1.

Patients were monitored every 2 cycles by ultrasound. After 6 cycles of chemotherapy all patients had surgery. In cases of clinical complete remission, the clips were wire-marked using mammographic control. Intraoperative specimen radiography was performed irrespective of the way of preoperative localization to detect the clip. Axillary dissection was performed if pre-therapeutic lymph node status was positive. pCR was defined as no invasive tumor in breast or lymph nodes after neoadjuvant treatment. Nodal conversion was defined as histologically negative lymph nodes after neoadjuvant therapy in patients with positive lymph nodes (SLNB or core-cut biopsy) at baseline. After surgery trastuzumab was continued $\mathrm{q} 3 \mathrm{w}$ up to 1 year. Radiotherapy was applied according to the standard in our center. All hormone receptor-positive patients received endocrine therapy. All postmenopausal patients received zoledronate $\mathrm{q} 6 \mathrm{~m}$ for 60 months. 


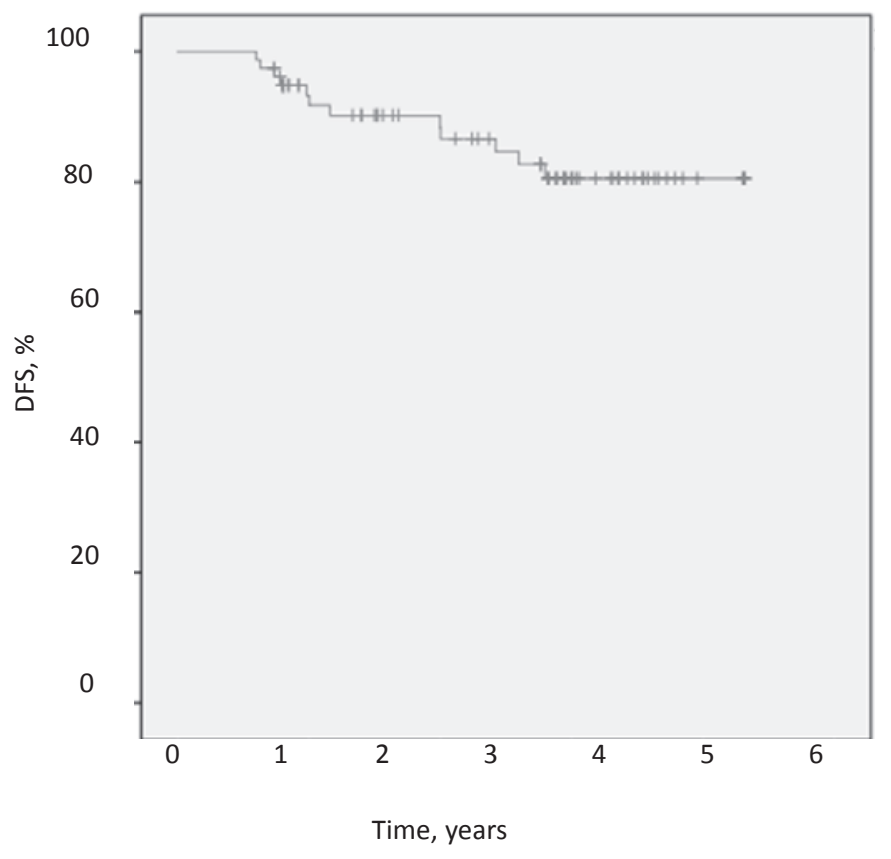

Fig. 1. Kaplan-Meier estimate for disease-free survival (DFS).

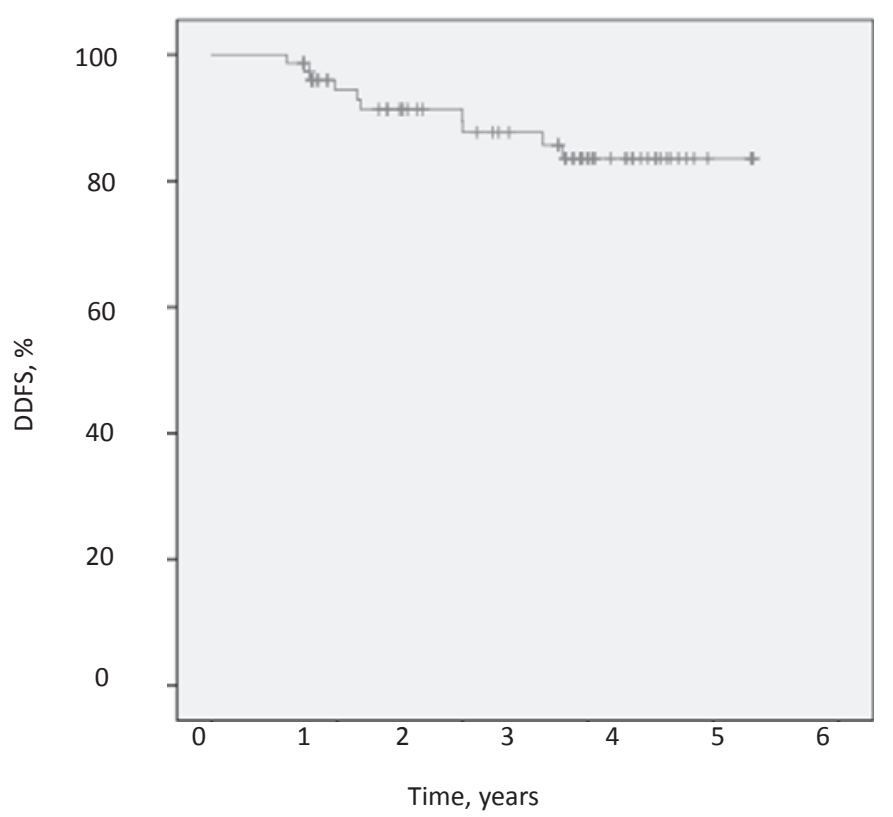

Fig. 2. Kaplan-Meier estimate for distant DFS (DDFS).

Toxicity was assessed every 3 -week cycle on therapy. Screening for congestive heart failure was performed by echocardiography at baseline, after 3 and 6 cycles and 12 months after surgery. Survival variables were collected from the database of our center where the complete follow-up of our patients is on file.

\section{Statistical Methods}

In this retrospective analysis we report the median disease-free survival (DFS, defined as local or distant recurrence or death from any cause), distant disease-free survival (DDFS, defined as distant recurrence or death from any cause) and the OS (defined as death from any cause) intervals after a median follow up of 48.5 months. Survival estimates were plotted using the Kaplan-

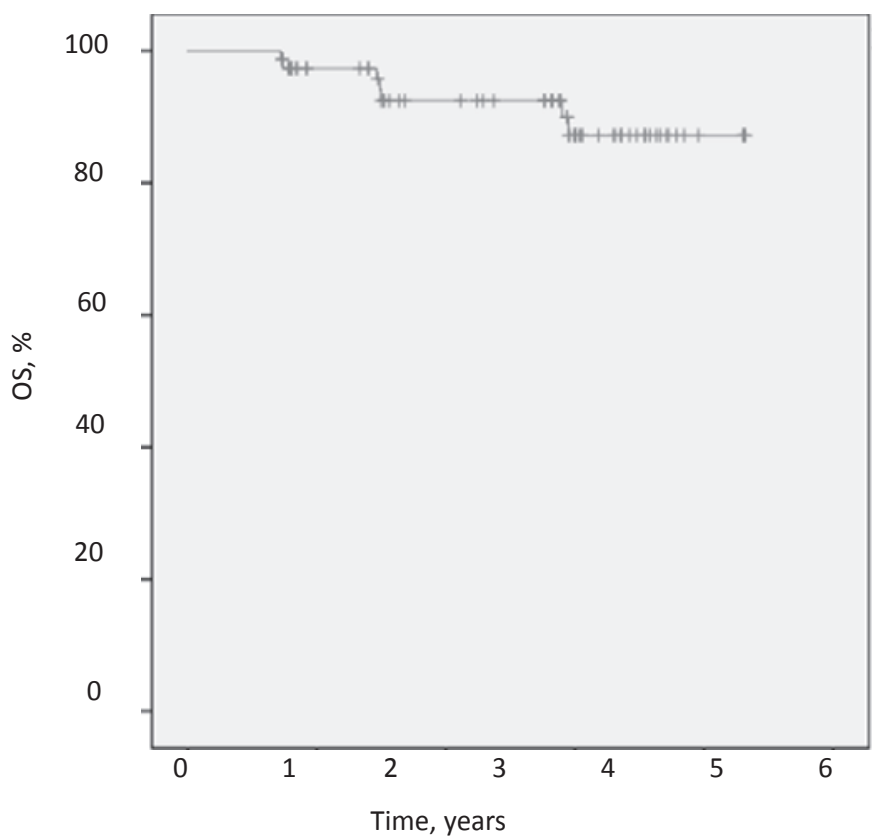

Fig. 3. Kaplan-Meier estimate for overall survival (OS).

Meier method. Correlation analysis between T status, hormone receptor status, lymph node status, grading, pCR, nodal conversion and survival variables was performed using a univariate semi-parametric Cox regression with SPSS Statistics software version 20.0.0. A 2 -sided $p$ value of $<0.05$ was considered significant.

\section{Results}

No grade III/IV toxicities occurred and no case of congestive heart failure was observed. Neither dose modifications nor dose delays were necessary. 34 of the 78 patients (43.6\%) achieved a pCR, 27 of the 40 node-positive patients (67.5\%) experienced nodal conversion.

After a median follow-up of 48.5 months the DFS was $84.6 \%$, the DDFS was $87.2 \%$ and the OS was $91 \%$. Figures $1-3$ demonstrate Kaplan-Meier curves for the 3 survival variables. Hormone receptor status, grading, menopausal status, nodal conversion and pCR had no significant influence on the survival variables, whereas T stage and nodal status at baseline were found to be significantly associated with survival estimates. Significant results for the subgroups are shown in table 2 .

\section{Discussion}

The dataset presented in this analysis represents the longest published follow-up to date for a cohort treated with neoadjuvant $\mathrm{TCH}$. Outcome in our analysis after a median follow up of 48.5 months compares well to outcome data observed in adjuvant trastuzumab trials looking at the trastuzumab-containing arms after the same or a shorter period of observation, such as the HERA 


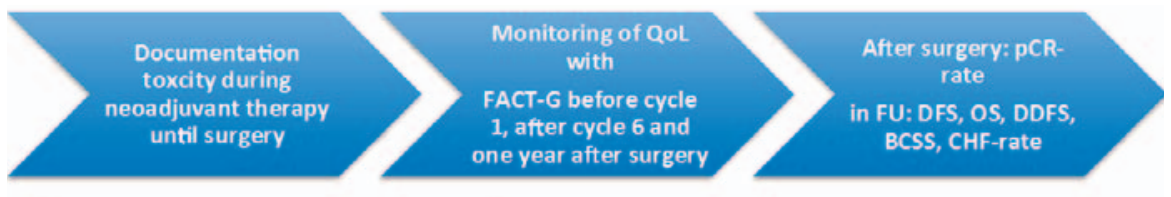

Fig. 4. Design of the FREE trial. Inclusion criteria: HER2neu-positive, non-metastatic breast cancer; tumor board recommendation for neoadjuvant therapy with docetaxel/carboplatin/trastuzumab/ pertuzumab.

\section{Co-primary endpoints: $\mathrm{pCR}$ and DFS}

All ER and/or PR positive patients are receiving an endocrine therapy. Adjuvant radiotherapy will be applied according to the current national guidelines. Trastuzumab will be completed up to 1 year after neoadjuvant therapy in all patients. Planned FU is 60 months. trial (4-year DFS 78.6\%, 4-year OS 89.3\%) [11], the BCIRG-006 trial (3-year DFS 82\%, 3-year OS 91\% in the TCH arm) [12] and the joint analysis of NCCTG N9831 and NSABP B-31 (4-year DFS 85.9\%, 4-year OS 92.6\%) [13].

The comparable efficacy of anthracycline-containing and anthracycline-free regimens in the adjuvant therapy of HER2-positive breast cancer has recently been demonstrated based upon the 10-year follow-up of the BCIRG-006 trial. In this trial, HRs for DFS for adriamycin/cyclophosphamide $\left(60 / 600 \mathrm{mg} / \mathrm{m}^{2} \mathrm{q} 3 \mathrm{w} \times 4\right)$ followed by docetaxel $\left(100 \mathrm{mg} / \mathrm{m}^{2} \mathrm{q} 3 \mathrm{w} \times 4\right)$ and trastuzumab $\times 1$ year $($ AC- $\mathrm{TH})$ or docetaxel/carboplatin $\left(75 \mathrm{mg} / \mathrm{m}^{2} /\right.$ AUC $\left.6 \mathrm{q} 3 \mathrm{w} \times 6\right)$ and trastuzumab $\times 1$ year $(\mathrm{TCH})$ were reported to be 0.70 and 0.76 , respectively, compared to the trastuzumab-free arm of the trial. HRs for OS were 0.64 and 0.76 for $\mathrm{AC}-\mathrm{TH}$ and $\mathrm{TCH}$, respectively, and were statistically significant in both arms. Of particular interest is the significant reduction of cardiotoxicity in the TCH arm compared to AC-TH in the long-term follow-up. The rate of symptomatic congestive heart failure events was 5 -fold lower following $\mathrm{TCH}(\mathrm{n}=4 ; 0.4 \%)$ compared to AC-TH ( $=21 ; 2.0 \%)$ $(\mathrm{p}=0.0005)$. The number of patients suffering from a left ventricular ejection fraction decline of $>10 \%$ after AC-TH $(\mathrm{n}=206)$ was double that after TCH $(n=97)(p<0.0001)[14]$. These data led to the establishment of $\mathrm{TCH}$ as 1 of the standards in the adjuvant treatment of HER2-positive breast cancer. Our data presented here support the use of this regimen also in the neoadjuvant setting.

Our statistical analysis could not identify any subgroup not benefitting from TCH. Although we have only reported significant associations of survival variables with tumor size and nodal status at baseline, the other analyses on hormone receptor status, grading, menopausal status, nodal conversion and pCR showed similar trends, but lacked a statistical significance in these analyses. This is probably being due to our comparably small cohort. The better outcome in patients with favorable prognostic parameters was not surprising and confirmed that the impact of prognostic parameters does not get lost during follow-up after neoadjuvant TCH.

In 2012, the results of the Neo Sphere trial showed a pCR rate of $45.8 \%$, establishing the combination of trastuzumab and pertuzumab with chemotherapy (in the trial monochemotherapy with docetaxel) as the new neoadjuvant standard therapy for HER2-pos- itive breast cancer [15]. The TRYPHAENA trial, published 1 year later, compared 2 anthracycline-containing regimens (5-fluorouracil, epirubicin, cyclophosphamide (FEC) + pertuzumab + trastuzumab followed by docetaxel + pertuzumab + trastuzumab; and FEC followed by docetaxel + pertuzumab + trastuzumab) to the anthracycline-free regimen docetaxel + carboplatin + pertuzumab + trastuzumab using the same chemotherapy backbone as TCH. pCR rates in the 2 anthracycline-containing arms were $61.6 \%$ and $57.3 \%$, respectively, whereas patients in the anthracycline-free arm achieved a pCR rate of $66.2 \%$ [16]. These data impressively underline the high efficacy of anthracycline-free regimens in the neoadjuvant therapy of HER2-positive breast cancer using a chemotherapy backbone containing of a taxane and a platinum salt. Based on the results of the TRYPHAENA trial, and encouraged by our results in the 4 year follow up of neoadjuvant $\mathrm{TCH}$, we are planning a follow-up trial on neoadjuvant TCPH (docetaxel/carboplatin/ pertuzumab and trastuzumab in analogy to the regimen used in the TRYPHAENA trial) that is momentarily in the process of achieving the approval of our ethical committee. The design of the trial is shown in figure 4.

In conclusion, the anthracycline-free $\mathrm{TCH}$ regimen is effective and safe in the neoadjuvant therapy of HER2-positive breast cancer, yielding DFS, DDFS and OS probabilities comparable to the results of adjuvant trials. Our data support the use of TCH as a neoadjuvant therapy regimen for patients with HER2-positive breast cancer. They also strongly encourage the use of taxanes and platinum salts as the chemotherapy backbone in studies investigating dual blockade with trastuzumab and pertuzumab in the neoadjuvant setting.

\section{Disclosure Statement}

Dr. Kolberg reports personal fees from Carl Zeiss meditec, Theraclion, Novartis, Amgen, Janssen, GSK, LIV Pharma, and Genomic Health. Dr. Akpolat-Basci, Dr. Stephanou and Dr. Aktas have nothing to disclose. Dr. Hannig reports personal fees from BMS, Boehringer Ingelheim, Novartis, Roche, Celgene, and Hexal. Dr. Liedtke reports personal fees from Celgene, TEVA, Pierre Fabre, Novartis, Amgen, Eisai, GSK, Roche, and Genomic Health. 


\section{References}

1 Piccart-Gebhart MJ, Procter M, Leyland-Jones B, et al Trastuzumab after adjuvant chemotherapy in HER2positive breast cancer. N Engl J Med 2005;353:16591672.

2 Slamon D, Eiermann W, Robert N, et al.: Adjuvant trastuzumab in HER2-positive breast cancer. N Engl J Med 2011;365:1273-1283.

3 Untch M, Rezai M, Loibl S, et al.: Neoadjuvant treatment with trastuzumab in HER2-positive breast cancer: results from the GeparQuattro study. J Clin Oncol 2010;28:2024-2031.

4 Cortazar P, Zhang L, Untch M, et al.: Pathological complete response and long-term clinical benefit in breast cancer: The CTNeoBC pooled analysis. Lancet 2014;384:164-172.

5 Chen W, He J, Song S, et al.: Efficacy of TCH/TEC neoadjuvant chemotherapy for the treatment of HER-2-overexpressing breast cancer. Oncol Lett 2015; 9:1922-1926.

6 Lin C, Chen DR, Chang KJ, et al.: A phase II study of neoadjuvant chemotherapy with docetaxel, cisplatin and trastuzumab for T2 breast cancers. Cancer Chemother Pharmacol 2012;69:1363-1368.

7 Sikov WM, Dizon DS, Strenger R, et al.: Frequent pathologic complete responses in aggressive stages II to III breast cancers with every-4-week carboplatin and weekly paclitaxel with or without trastuzumab: A Brown University Oncology Group Study. J Clin Oncol 2009;27:4693-4700.
8 Shinde AM, Zhai J, Yu KW, et al: Pathologic complete response rates in triple-negative, HER2-positive, and hormone receptor-positive breast cancers after anthracycline-free neoadjuvant chemotherapy with carboplatin and paclitaxel with or without trastuzumab. Breast 2015;24:18-23.

9 Kolberg HC, Akpolat-Basci L, Otterbach F, et al.: Docetaxel, carboplatin and weekly trastuzumab are active as neoadjuvant therapy in operable HER2-positive breast cancer. Breast 2011;20 (Suppl 1):S74.

10 Bayraktar S, Gonzalez-Angulo AM, Lei X, et al.: Efficacy of neoadjuvant therapy with trastuzumab concurrent with anthracycline- and nonanthracycline-based regimens for HER2-positive breast cancer. Cancer 2012;118:2385-2393.

11 Gianni L, Dafni U, Gelber RD, et al.: Treatment with trastuzumab for 1 year after adjuvant chemotherapy in patients with HER2-positive early breast cancer: A 4-year follow-up of a randomised controlled trial. Lancet Oncol 2011;12:236-244.

12 Slamon D, Eiermann W, Robert N, et al.: BCIRG 006: 2nd interim analysis phase III randomized trial comparing doxorubicin and cyclophosphamide followed by docetaxel (AC-T) with doxorubicin and cyclophosphamide followed by docetaxel and trastuzumab (AC-TH) with docetaxel, carboplatin and trastuzumab (TCH) in Her2neu positive early breast cancer patients. Breast Cancer Res Treat 2006;95:S52.
13 Perez EA, Romond EH, Suman VI, et al.: Updated results of the combined analysis of NCCTG N9831 and NSABP B-31 adjuvant chemotherapy with/without trastuzumab in patients with HER2-positive breast cancer. J Clin Oncol 2007;25 (Suppl):512

14 Slamon DJ, Eiermann W, Robert NJ, et al.: Ten year follow-up of BCIRG-006 comparing doxorubicin plus cyclophosphamide followed by docetaxel $(\mathrm{AC} \rightarrow \mathrm{T})$ with doxorubicin plus cyclophosphamide followed by docetaxel and trastuzumab $(\mathrm{AC} \rightarrow \mathrm{TH})$ with docetaxel, carboplatin and trastuzumab (TCH) in HER2+ early breast cancer. Cancer Res 2015;76 (Suppl):S5-04.

15 Gianni L, Pienkowski T, Im YH, et al.: Efficacy and safety of neoadjuvant pertuzumab and trastuzumab in women with locally advanced, inflammatory, or early HER2-positive breast cancer (NeoSphere): A randomised multicentre, open-label, phase 2 trial. Lancet Oncol 2012;13:25-32.

16 Schneeweiss A, Chia S, Hickish T, et al.: Pertuzumab plus trastuzumab in combination with standard neoadjuvant anthracycline-containing and anthracyclinefree chemotherapy regimens in patients with HER2 positive early breast cancer: A randomized phase II cardiac safety study (TRYPHAENA). Ann Oncol 2013; 24:2278-2284. 